of unconventional faults in CMOS circuits, "IEEE Trans. ComputerAided Design, vol. 10, pp. 667-682, May 1991.

[6] R. Lisanke, "Logic synthesis and optimization benchmarks user guide, version 2.0," Microelectronics Center of North Carolina, Tech. Rep.

[7] P. Cox, P. Yang, S. Mahant-Shetti, and P. Chatterjee, "Statistical modeling for efficient parametric yield estimation of MOS VLSI circuits," IEEE J. Solid-State Circuits, vol, SC-20, pp. 391-398. 1985.

[8] K. Wagner, C. Chin, and E. McCluskey, "Pseudorandom testing," IEEE Trans. Computers, vol. 36. pp. 333-343, 1987

\section{On Performance Bounds for a Class of Rectilinear Steiner Tree Heuristics in Arbitrary Dimension}

Andrew B. Kahng and Gabriel Robins

\begin{abstract}
We give a family of examples on which a large class $C$ of "minimum spanning tree-based" rectilinear Steiner tree heuristics has performance ratio arbitrarily close to $3 / 2$ times optimal. The class $C$ contains many published heuristics whose worst-case performance ratio: were previously unknown. Of particular interest is that $C$ contains two heuristics whose worst-case ratios had been conjectured to be bounded away from $3 / 2$, and our construction also points out an incorrect claim of optimality for one of these heuristics. Our examples also force worst-possible behavior in a number of heuristics outside $C$. The construction generalizes to $d$ dimensions, where the heuristics wil have performance ratio of at least $(2 d-1 / d)$; this improves the previous lower bound on performance ratio in arbitrary dimension.
\end{abstract}

\section{INTRODUCTION}

The minimum rectilinear Steiner tree (MRST) problem has been extensively studied in VLSI layout because solutions correspond to optimal circuit wiring in the $L_{1}$ (Manhattan) norm. The problem is defined as follows

The MRST Problem: Given a set $P$ of points in the $L_{1}$ plane, determine a set $S$ of Steiner points such that the minimum spanning tree on $P \cup S$ has minimum cost.

Here, the cost of a tree is the sum of the costs ( $L_{l}$ lengths) of its edges. The MRST problem is NP-complete [5], and a number of heuristics have been proposed. Hwang [9] showed that the rectilinear minimum spanning tree (MST) is itself an approximation to the MRST with worst-case ratio $\operatorname{cost}(\mathrm{MST}) / \operatorname{cost}(\mathrm{MRST}) \leq 3 / 2$. This result, along with efficient methods for computing the MST of a planar point set, has motivated a number of MRST heuristics which start with an MST construction and then improve the solution by various methods (e.g., overlapping edges to induce Steiner points). Instances of this approach include the work of Hasan, $\mathrm{Vi}$ jayan, and Wong [7], Ho, Vijayan, and Wong [8], Hwang [11], Lee, Bose, and Hwang [14], and Lee and Sechen [15]. Other heuristics, such as those discussed by Bern [1], Bern and Carvalho [2], Richards [17], and Servit [18], emulate the classic MST construc

Manuscript received April 10, 1990; revised September 23, 1991. This work was supported in part by the National Science Foundation under Gran MIP-9110696 and by an IBM Graduate Fellowship. This paper was recommended by Associate Editor M Marek-Sadowska.

A. B. Kahng is with the Department of Computer Science, University of California, Los Angeles, CA 90024.

G. Robins was with the Department of Computer Science, University of California, Los Angeles. He is now with the Department of Computer Science, University of Virginia, Charlottesville, VA 22903.

IEEE Log Number 9200530 tions of Kruskal and Prim [13], [16] while building the Steiner tree As noted by Richards [17] and in such surveys as those of Hwang $[10]$ and Winter [20], these methods yield very similar results on random instances, i.e., heuristic Steiner tree cost $8 \%$ to $9 \%$ less than MST cost on average.

Since these Steiner tree constructions cannot have greater cost than the minimum spanning tree, the bound of $3 / 2$ proved by Hwang is a trivial upper bound on the worst-case performance ratio of these heuristics. However, the actual performance ratios for many "MST-based" methods have remained unknown. At times there has been hope that certain methods might be provably better than the simple MST approximation (e.g., [12]; the algorithms of Bern [1.] and Ho, Vijayan, and Wong [8] are two more recent examples).

This paper shows that any Steiner tree heuristic in a very general class $C$ of "MST-based" methods will have worst-case performance ratio arbitrarily close to $3 / 2$, i.e., the same bound as for the MST itself. This result is based on two simple constructions. We then show that many published heuristics [1], [2], [7], [8], [12], [17], [18] with previously unknown worst-case behavior fall into the class $C$, and thus we simultaneously resolve a number of error bounds. Our construction also points out a recent incorrect claim in [8] that the two heuristics of [8] yield optimal Steiner trees on a certain class of inputs. Furthermore, our examples also establish a lower bound of $3 / 2$ on performance ratios for other heuristics which are not in the class $C$, e.g., [12], [14], [19]. Finally, the examples generalize to $d$ dimensions, where all of these heuristics will have error bound of at least $(2 d-1) / d$. The previous lower bound on the performance ratio was $2(d-1) / d$ [4], [6]. From these results, it seems doubtful that the popular MST-based approach will ever afford a better worst-case ratio than the simple MST bound of $3 / 2$.

\section{Counterexamples for Two Dimensions}

We begin this section by discussing two common approaches to constructing a heuristic Steiner tree in the Manhattan plane. We exhibit pathological examples for these methods and then show that the same instances will force a $3 / 2$ performance ratio for an entire class $C$ of Steiner tree constructions.

The first popular approach to the MRST problem starts with a rectilinear MST and computes a Steiner tree by "overlapping", edges of the MST as much as possible, as shown in Fig. 1. Clearly, the result cannot have cost greater than the MST cost. A general template for this MST-Overlap heuristic is as follows:

\section{MST-Overlap:}

Input: fixed rectilinear MST

Determine a shorter Steiner tree which lies completely within the union of bounding boxes of the MST edges.

A number of authors have explored this idea, including Hwang [11]. Lee, Bose, and Hwang [14] and Lee and Sechen [15]. Ho, Vijayan, and Wong [8] recently gave the surprising result that the optimal RST derivable in this fashion can be computed in linear time; thus, their method is strictly better than those of [11], [14], and [15]. Several researchers conjectured that the worst-case performance ratio of the new method in $[8]$ was less than $3 / 2$. However, the example of Fig. 2 forces a sharp performance bound of exactly $3 / 2$

Note that the authors of [8] define a separable MST to be one whose edge bounding boxes do not intersect except at their borders, 

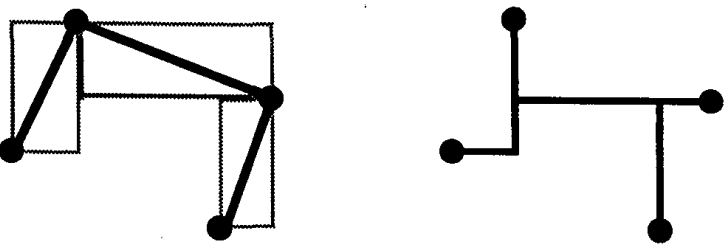

Fig. 1. Optimal overlap of MST edges within their bounding boxes.

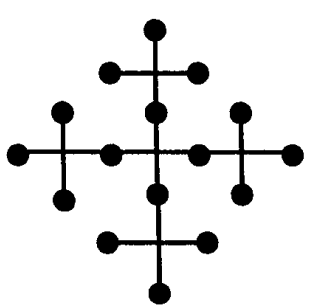

(a)

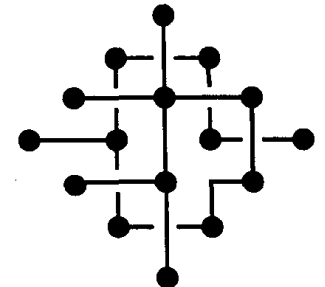

(b)
Fig. 2. An example where the strict equality cost (MST-Overlap) $/ \operatorname{cost}($ MRST $)=3 / 2$

holds. (a) MRST (cost $=20$ ); (b) any Steiner tree derived from this MST will have cost $=30$.

and their linear-time algorithm actually finds optimal overlaps for separable initial MSTs (the MST of Fig. 2 is not separable). However, even when we insist that the starting MST be separable, we can still force a performance ratio arbitrarily close to $3 / 2$, as illustrated in Fig. 3. Fig. 3(a) shows a separable MST on a pointset where the strict equality cost (MST) $/ \operatorname{cost}(\mathrm{MRST})=3 / 2$ holds; Fig. 3(b) shows a perturbation of the pointset such that the MST is unique; and Fig. 3(c) shows the optimal Steiner tree topology for both cases.

The example of Fig. 3(a) is separately included since it points out a misstatement [8, p. 192]: "Both the algorithms produce the optimum Steiner trees for each member of the class of point sets whose optimal RST has a cost which is $2 / 3$ that of the cost of the MST." This refers to the so-called S-MST and L-MST algorithms, which rely on the separable-MST (SMST) construction in $[8, \mathrm{p}$. 187]. It is straightforward to verify that on the pointset shown in Fig. 3(a), the tie-breaking rules of the SMST construction in [8] will force the initial separable MST to be exactly that shown in the figure. Edge overlapping improves this only marginally to the solution shown in Fig. 3(d), implying a performance ratio arbitrarily close to $3 / 2$, even though the optimal RST indeed has a cost exactly $2 / 3$ that of the MST.

Fig. 3 also shows that a "folklore" heuristic and its variants, described in [17] and ascribed to Clark Thompson by Bern [1], [2], has worst-case performance ratio arbitrarily close to $3 / 2$. We refer to this second generic type of construction as the Kruskal-Steiner heuristic, since it is an analog of Kruskal's MST construction [13]:

\section{Kruskal-Steiner:}

Input: $n$ isolated components (points)

Until one component remains, connect the closest pair of components.

Variants in the literature differ mostly in their definitions of "closest pairs" of components, but the example of Fig. 3(b) is immune to these distinctions. When any variant of Kruskal-Steiner is executed on the pointset of Fig. 3(b), it will start at the left end and alternate between the middle, top, and bottom rows, adding a

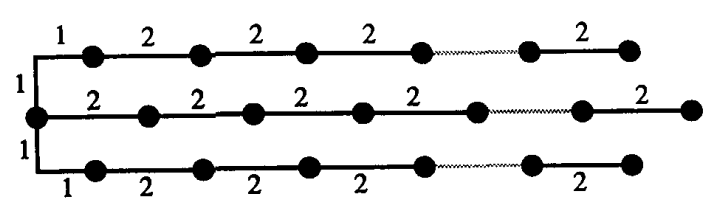

(a)

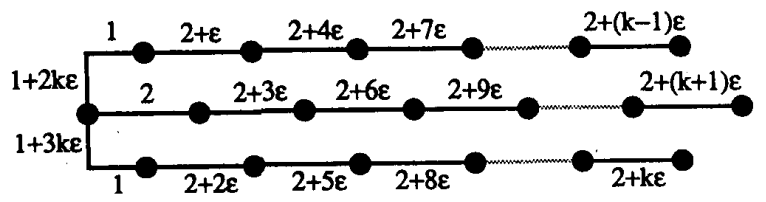

(b)

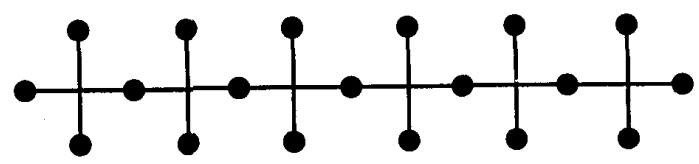

(c)

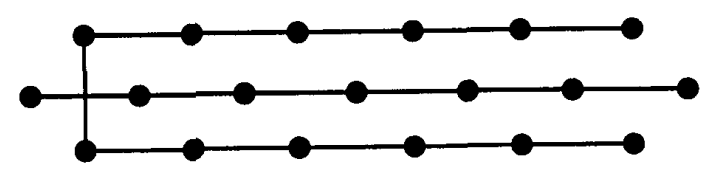

(d)

Fig. 3. An example of a separable MST where cost(MST-Overlap)/ cost (MRST) is arbitrarily close to $3 / 2$. For $n$ points, any Steiner tree derivable from the separable MSTs of (a) or (b) will have cost $2(n-2)$, while the MRST (c) has cost $(4 / 3) \cdot(n-1)$, yielding a performance ratio arbitrarily close to $3 / 2$ for large enough $n$. In (d), we show the best possible RST that can be produced by any MST-Overlap or Kruskal-Steiner heuristic.

single horizontal segment to each in turn. Therefore, the Steiner tree will consist entirely of straight horizontal line segments, except at the starting left end, and its cost will be arbitrarily close to $3 / 2$ times optimal. Note that the $\epsilon$ perturbations in Fig. 3(b) force the alternation between rows and make the heuristic construction completely deterministic.

The MST-overlap and Kruskal-Steiner heuristics form part of a very general class $C$ of greedy Steiner tree methods. We now define the class $C$ and show that the example of Fig. 3 is pathological for the entire class $C$.

Recall that the heuristic Steiner tree is a minimum spanning tree on the union of an input pointset $P$ and a set of Steiner points $S$. We define an edge as any wire connecting two points in $P \cup S$. The following terminology is used to denote progressively more general connection types: 1) a point-point connection is an edge between two points of $P ; 2$ ) a point-edge connection is a wire between a point of $P$ and an edge, inducing up to one Steiner point; and 3) an $e d g e-e d g e$ connection is a wire between two edges, which may induce up to two Steiner points.

We say that a greedy algorithm iteratively selects the best alternative from among all available alternatives. In the following definition, the iterative algorithms in $C$ are greedy with respect to Manhattan edge length. The class $C$ is defined as follows:

Heuristic $H \in C$

Input: $n$ isolated components (points of $P$ )

While there is more than one connected component do:

Select a connection type (point-point, point-edge, or edgeedge); 
Connect the closest pair of components greedily with respect to this connection type;

Optionally at any time, reroute any edge within its bounding box;

Optionally at any time, eliminate any edge overlap.

Theorem 1: Any heuristic in the class $C$ will have performance ratio arbitrarily close to $3 / 2$.

Proof: The MST of the pointset depicted in Fig. 3(b) is clearly unique, since all interpoint distances of length $<3$ are unique. Even if general connection types are allowed, all connections in the MST will be simple horizontal point-point connections except for exactly two connections, from the top row to the middle row and from the middle row to the bottom row. The greedy routing of every edge but these two is unique, since all edges except these two have degenerate bounding boxes. Note that no improvement is possible by edge rerouting within these degenerate edge bounding boxes. Therefore, no heuristic in $C$ can do better than the result depicted in Fig. 3(d). Since the effect of the optional rerouting of the two nondegenerate connections becomes negligible as the pointse grows large, the performance ratio is arbitrarily close to $3 / 2$. $\square$

We now list a number of published heuristics with previously unknown performance ratio, all of which are shown by Theorem 1 to have error bounds arbitrarily close to $3 / 2$. We do not reproduce the various authors" descriptions of each algorithm that we mention here, since it is very easy to see from the high-level classification that these algorithms are indeed in $C$. Algorithms which follow a greedy Kruskal-type construction satisfy the verbatim definition of the class $C$ : these include the methods of Hwang [11] and Lee and Sechen [15], in addition to methods described in Bern [1], [2], Richards [17], and Servit [18]. It is also easy to see that algorithms which start with an initial MST and then overlap rectilinear edges within their bounding boxes, such as those of Hasan, Vijayan, and Wong [7] and Ho, Vijayan, and Wong [8], are members of $C$, since using only point-point connections will build an MST, and the optional rerouting is then used to induce edge overlaps. Interestingly, exponential-time methods can also fall into the class $C$, e.g., the suboptimal branch-and-bound method of Yang and Wing [21]. Theorem 1 implies that all of these methods have the same worstcase error bound as the simple MST.

Finally, the counterexample of Fig. 3 also establishes new lower bounds arbitrarily close to $3 / 2$ for the performance ratios of several heuristics not in $C$, such as the three-point connection methods of Hwang [12] and Lee, Bose, and Hwang [14], and the Delaunay triangulation-based method of Smith, Lee, and Liebman [19]. This is easy to verify using the pointset in Fig. 3(b): as with the heuristics in $C$, these latter methods are severely constrained by the nature of the unique minimum spanning tree.

We note that the recent work of De Souza and Ribiero [3] constructs a similar instance to that of Fig. 3 and also discusses the worst-case performance of RST heuristics. However, the work of [3] is limited to two dimensions, while Section III below extends our construction to yield new bounds in higher dimensions. More importantly, the work of De Souza and Ribiero is concerned solely with several specific algorithms and thus does not establish a general result as we do in Theorem 1.

\section{Extensions to Higher Dimensions}

Most rectilinear Steiner tree heuristics, including the MST-Overlap and Kruskal-Steiner variants, extend to higher dimensions and are of special interest for emerging multilayer packaging and "three-dimensional" process technologies. However, the exam-

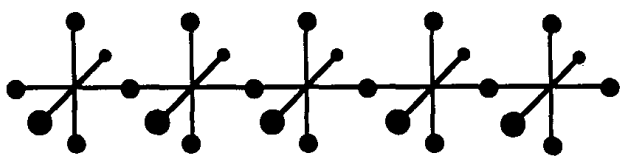

(a)

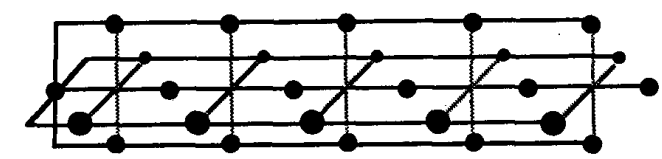

(b)

Fig. 4. For $d=3$, the MRST (a) has cost $6 / 5 \cdot(n-1)$, while any MRST derivable from the MST ( b) has cost $2(n-3)$, yielding performance ratio arbitrarily close to $5 / 3$ for $n$ large.

ples of Figs. 2 and 3 also generalize to $d$ dimensions and provide new lower bounds on the performance ratio of heuristics in $C$. In particular, the example of Fig. 3 generalizes to $n=(2 d-1) k+$ 1 points for any given positive integer $k$ : the cost of the optimal Steiner tree is at most $2 d(n-1) /(2 d-1)$, the cost of the (unique, separable) MST is $2(n-1)$, and the cost of the best Steiner tree derivable from this MST is $2(n-d)$, as illustrated in Fig. 4 for $d$ $=3$. Thus, in $d$ dimensions the performance ratio of a heuristic in class $C$ will be arbitrarily close to $(2 d-1) / d$. This value improves the lower bound for the worst-case MST/MRST ratio in higher dimensions from the previously known value of $2(d-1) / d[4]$, [6].

\section{CONCLUSIONS}

We conjecture that $(2 d-1) / d$ is not only a lower bound, but also a general upper bound for the worst-case performance ratio in $d$ dimensions of any MRST heuristic in $C$. Thus, $(2 d-1) / d$ would be the higher dimensional analogue of Hwang's value of $3 / 2$ for two dimensions. The basic question remains whether there is an MRST heuristic with worst-case performance ratio bounded away from $3 / 2$. The result of Theorem 1 suggests that the "MST-based" approach, which has dominated the VLSI Steiner routing literature, is not likely to yield such a bound.

\section{ACKNOWLEDGEMENTS}

The authors thank the anonymous referees for their dedication and instructive comments. They are particularly grateful to Reviewer 3 for pointing out the related work of de Souza and Ribiero.

\section{REFERENCES}

[1] M. W. Bern, "Two probabilistic results on rectilinear Steiner trees," Algorithmica, vol. 3, pp. 191-204, 1988.

[2] M W Bern, and M. De Carvalho "A greedy heuristic for the rectilinear Steiner tree problem," Computer Science Division (EECS), University of California, Berkeley, Rep. UCB/CSD 87/306, 1986.

[3] C. C. de Souza and C. C. Ribiero, "A tight worst case bound for the performance ratio of heuristics for the minimum rectilinear Steiner tree problem." OR Spektrum, vol. 12, pp. 109-111, 1990.

[4] L. R. Foulds, "Maximum Savings in the Steiner Problem in Phylogeny," J. Theoretical Biol., vol. 107, pp. 471-474, 1984.

[5] M. Garey and D. S. Johnson, "The rectilinear Steiner problem is NP-complete," SIAM J. Applied Math., vol. 32, no. 4, pp. 826-834, April 1977

[6] E. N. Gilbert and H. O. Pollak, "Steiner Minimal Trees," SIAM J. Applied Math., vol. 16, pp. 1-29, Jan. 1968

[7] N. Hasan, G. Vijayan, and C. K. Wong, "A neighborhood improve- 
ment algorithm for rectilinear Steiner trees," in Proc. IEEE Intl. Symp. Circ. and Sys., New Orleans, 1990, pp. 1-6.

[8] J.-M. Ho, G. Vijayan, and C. K. Wong, "New algorithms for the rectilinear Steiner tree problem," IEEE Trans. Computer-Aided Design, vol. 9, pp. 185-193, Feb. 1990.

[9] F. K. Hwang, "On Steiner minimal trees with rectilinear distance," SIAM J. Applied Math., vol. 30, no. 1, pp. 104-114, Jan. 1976

[10] F. K. Hwang, "The rectilinear Steiner problem,"' J. Design Automat. and Fault-Tolerant Comp., vol. 2, pp. 303-310, 1978

[11] F. K. Hwang, "An $O(n \log n)$ algorithm for rectilinear minimal spanning trees," J. Ass. Comput. Mach., vol. 26, no. 2, pp. 177-182, Feb. 1979.

[12] F. K. Hwang, "An $O(n \log n)$ algorithm for suboptimal rectilinear Steiner trees," IEEE Trans. Circuits Syst., vol. CAS-26, pp. 75-77, Jan. 1979

[13] M. Kruskal, "On the shortest spanning subtree of a graph, and the traveling salesman problem," in Proc. AMS, vol. 7, 1956, pp. $48-50$.

[14] J. H. Lee, N. K. Bose and F. K. Hwang, "Use of Steiner's problem in sub-optimal routing in rectilinear metric," IEEE Trans. Circuits Syst., vol. CAS-23, pp. 470-476, 1976.

[15] K. W. Lee and C. Sechen, "A new global router for row-based layout," in Proc. IEEE Int. Conf. on Computer-Aided Design, Santa Clara, CA, Nov. 1988, pp. 180-183.

[16] A. Prim, "Shortest Connecting Networks and Some Generalizations," Bell Syst. Tech J., vol. 36, pp. 1389-1401, 1957.

[17] D. Richards, "Fast heuristic algorithms for rectilinear Steiner trees," Algorithmica, vol. 4, pp. 191-207, 1989.

[18] M. Servit, "Heuristic Algorithms for Rectilinear Steiner Trees," Digital Process., vol. 7, no. 1, pp. 21-31, 1981.

[19] J. M. Smith, D. T. Lee, and J. S. Liebman, "An $O(N \log N)$ heuristic algorithm for the rectilinear Steiner minimal tree problem," Engineering Optimization, vol. 4, pp. 179-192, 1980.

[20] P. Winter, "Steiner problem in networks: a survey," Networks, vol. 17, pp. 129-167, 1987.

[21] Y. Y. Yang and O. Wing, "Suboptimal algorithm for a wire routing problem," IEEE Trans. Circuit Theory, vol. CT-19, pp. 508-511, 1972. 